\title{
Communication
}

\section{Direct Measurement of Lateral Force Using Dual Cantilevers}

\section{Makoto Ishikawa *, Masaya Ichikawa and Kouji Miura}

Department of Physics, Aichi University of Education, Hirosawa 1, Igaya-cho, Kariya 448-8542, Japan; E-Mails: s2080879@auecc.aichi-edu.ac.jp (M.I.); kmiura@auecc.aichi-edu.ac.jp (K.M.)

* Author to whom correspondence should be addressed; E-Mail: makoishi@auecc.aichi-edu.ac.jp.

Received: 27 January 2012; in revised form: 29 February 2012 / Accepted: 2 March 2012 /

Published: 7 March 2012

\begin{abstract}
We have constructed an experimental system to measure a piconewton lateral force using dual cantilevers which cross with each other. The resolution of the lateral force is estimated to be $3.3 \mathrm{p} \pm 0.2 \mathrm{pN}$, which is comparable to forces due to thermal fluctuation. This experimental apparatus works so easily that it will enable us to determine forces during nano-manipulation and nano-tribological measurements.
\end{abstract}

Keywords: lateral force; dual cantilevers; piconewton

\section{Introduction}

Measurements of lateral force using scanning probe microscopy have been of great importance in nanoscience and nanotribology [1], because it is necessary to know force needed to move small objects on a surface and to obtain friction force on an atomic scale [2]. However, it is difficult to obtain a correct lateral force by using the conventional lateral force microscope (LFM) because it generally uses the torsion of a micro-cantilever to measure lateral force [3-6]. Here, we propose a novel method to obtain a lateral force by using dual cantilevers which cross with each other.

\section{Experimental Section}

The overview of experimental setup is depicted in Figure 1(a). This novel setup consists of a 3D-PZT driven actuator (Unisoku Co. Ltd., Japan) and a self-detecting cantilever (NPX1CTP003, SII) as a force sensor on the commercial AFM (SPI-3000, Seiko Instrument, Japan). The self-detecting cantilever attached to the 3D-PZT driven actuator is fixed to the cantilever holder and optical head of the AFM using a C-shaped jig. The 3D-PZT driven actuator has two modes of inertia driven motion 
and an extension of the 3D-PZT block, which perform the coarse and fine movements for any direction, respectively. The maximum traveling length of the inertia driven motion is $2 \mathrm{~mm}$. The self-detecting cantilever has PZT resistors which act as a small strain gauge. Thus, the force acting on the self-detecting cantilever can be changed into the resistance by supplying the voltage to the bridge circuit, the details of which have been described in a previous paper [7]. The cantilever on the AFM and the self-detecting cantilever are fixed perpendicular to each other so that the bending direction of the self-detecting cantilever is arranged parallel to the torsional direction of the cantilever on AFM, as illustrated in Figure 1(b), where the edge of the self-detecting cantilever comes into contact with the top of the cantilever tip on the AFM. Thus, the bending force acting on the self-detecting cantilever is the same as the lateral force acting on the tip on AFM. The signals of normal and lateral forces acting on the cantilever on the AFM are output from the monitor terminals on the AFM system. On the other hand, another computer controls the movement of the self-detecting cantilever and detects the output signal from the self-detecting cantilever. The experimental apparatus works so easily that its performance is excellent.

Figure 1. (a) Schematic of experimental apparatus. (b) Cantilever on AFM and self-detecting cantilever arranged perpendicular to each other.

(a)

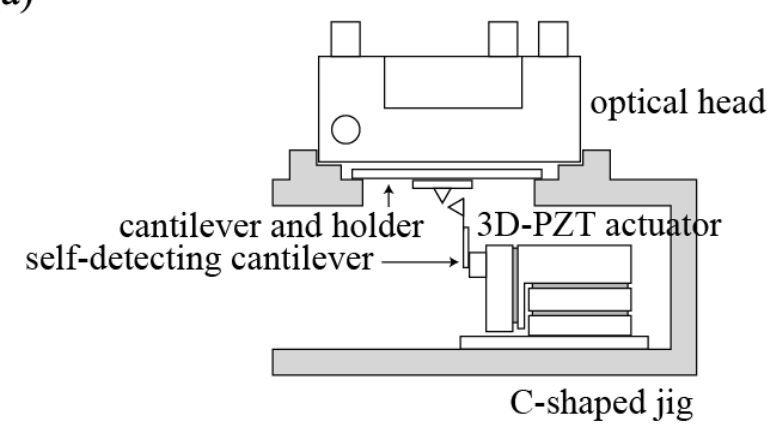

(b)

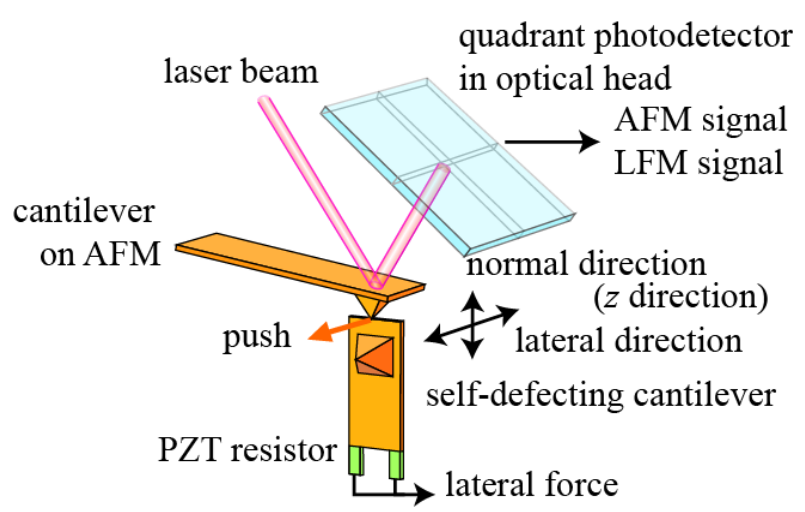

\section{Results}

First, the self-detecting cantilever is moved to the bottom of the cantilever tip on the AFM by using the inertia driven motion of the 3D-PZT driven actuator under the optical microscope to check the experimental setup. The self-detecting cantilever is carefully moved up until the signal of normal force from the cantilever on the AFM changes. After that, the self-detecting cantilever is driven up and down by using the extension of the PZT block to measure the sensitivity along the normal direction ( $\mathrm{z}$ direction) of the self-detecting cantilever. Figure 2 shows the AFM signal versus the traveling length along the $\mathrm{z}$ direction of the self-detecting cantilever, which clearly exhibits their proportional relationship. The sensitivity was estimated to be $7.5 \mathrm{mV} / \mathrm{nm}$ from the slope of Figure 2, which is consistent with one obtained by using the AFM. Hence it is confirmed that the movement along the $\mathrm{z}$ direction of the self-detecting cantilever works well. 
Figure 2. AFM signal vs. traveling length along $z$ direction.

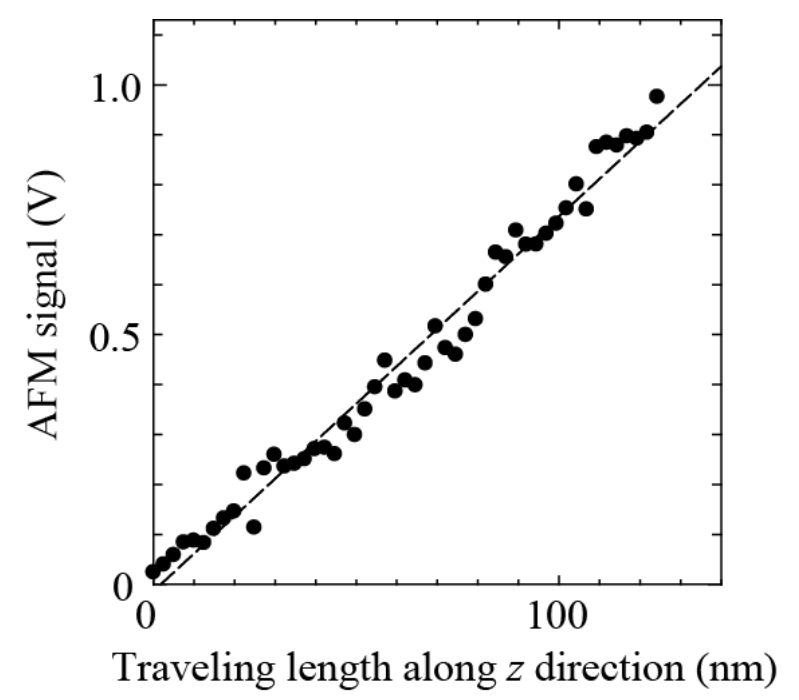

Figure 3. LFM signal vs. lateral force.

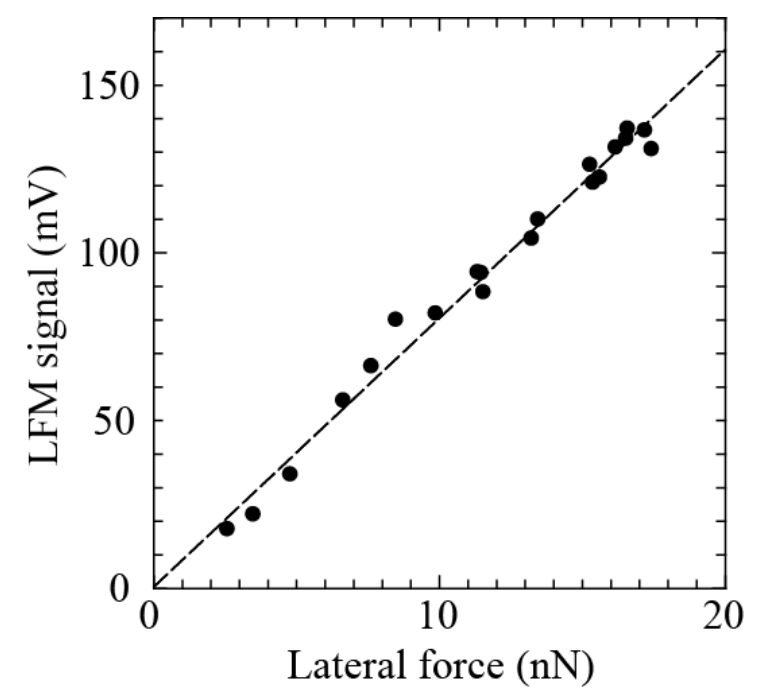

Next, the self-detecting cantilever is driven along the lateral direction of the cantilever on the AFM while monitoring the output of the LFM signal. Figure 3 shows the LFM signals versus lateral force, which clearly exhibits their proportional relationship. The sensitivity was estimated to be $8.0 \mathrm{mV} / \mathrm{nN} \pm 0.6 \mathrm{mV} / \mathrm{nN}$ from the slope of Figure 3, which is comparable to that presented by Schwarz et al. [8]. Thus, the resolution of the lateral force can be estimated to be $3.3 \mathrm{pN} \pm 0.2 \mathrm{pN}$ because the resolution of the LFM signal is $26.67 \mu \mathrm{V}$, which is comparable to forces due to thermal fluctuation. Hence it is found that this method has the potential to measure a lateral force of piconewtons.

\section{Conclusions}

In this paper, we have proposed the novel method to detect a piconewton lateral force using dual cantilevers which cross with each other. This experimental apparatus works so easily that its performance is excellent. This novel method will enable us to determine forces during nano-manipulation and nano-tribological measurements. 


\section{Acknowledgments}

This work was supported by the Grant-in-Aid for Challenging Exploratory Research (No. 21654041), Scientific Research (C) (No. 23560022).

\section{References}

1. Meyer, G.; Amer, N.M. Simultaneous measurement of lateral and normal forces with an optical-beam-deflection atomic force microscope. Appl. Phys. Lett. 1990, 57, 2089-2091.

2. Miura, K.; Tsuda, D.; Itamura, N.; Sasaki, N. Superlubricity of fullerene intercalated graphite composite. Jpn. J. Appl. Phys. 2007, 46, 5269-5274.

3. Sader, J.E.; White, L. Theoretical analysis of the static deflection of plates for atomic force microscope applications. J. Appl. Phys. 1993, 74, 1-9.

4. Neumeister, J.M.; Ducker, W.A. Lateral, normal, and longitudinal spring constants of atomic force microscopy cantilevers. Rev. Sci. Instrum. 1994, 65, 2527-2538.

5. Ogletree, D.F.; Carpick, R.W.; Salmeron, M. Calibration of frictional forces in atomic force microscop. Rev. Sci. Instrum. 1996, 67, 3298-3306.

6. Feiler, A.; Attard, P.; Larson, I. Calibration of the torsional spring constant and the lateral photodiode response of frictional force microscopes. Rev. Sci. Instrum. 2000, 71, 2746-2750.

7. Ishikawa, M.; Harada, R.; Sasaki, N.; Miura, K. Visualization of nanoscale peeling of carbon nanotube on graphite. Appl. Phys. Lett. 2008, 98, 083122.

8. Schwarz, U.D.; Köster, P.; Wiesendanger, R. Quantitative analysis of lateral force microscopy experiments. Rev. Sci. Instrum. 1996, 67, 2560-2567.

(C) 2012 by the authors; licensee MDPI, Basel, Switzerland. This article is an open access article distributed under the terms and conditions of the Creative Commons Attribution license (http://creativecommons.org/licenses/by/3.0/). 